\title{
Wie wirkt sich die Corona- Pandemie auf Angst- und Zwangsstörungen aus?
}

\author{
Margarete Stöcker
}

Bei Menschen, die schon vor dem Ausbruch der Corona-Pandemie an einer Angst- oder Zwangsstörung erkrankt sind, kann das Thema Sars-CoV-2 die Symptome verschlimmern: Sie verlassen aus Angst vor einer Ansteckung ihre Wohnung nicht mehr oder waschen sich zwanghaft die Hände. Unsere Autorin erklärt die unterschiedlichen Krankheitsbilder und Therapieansätze und veranschaulicht diese anhand eines Fallbeispiels.

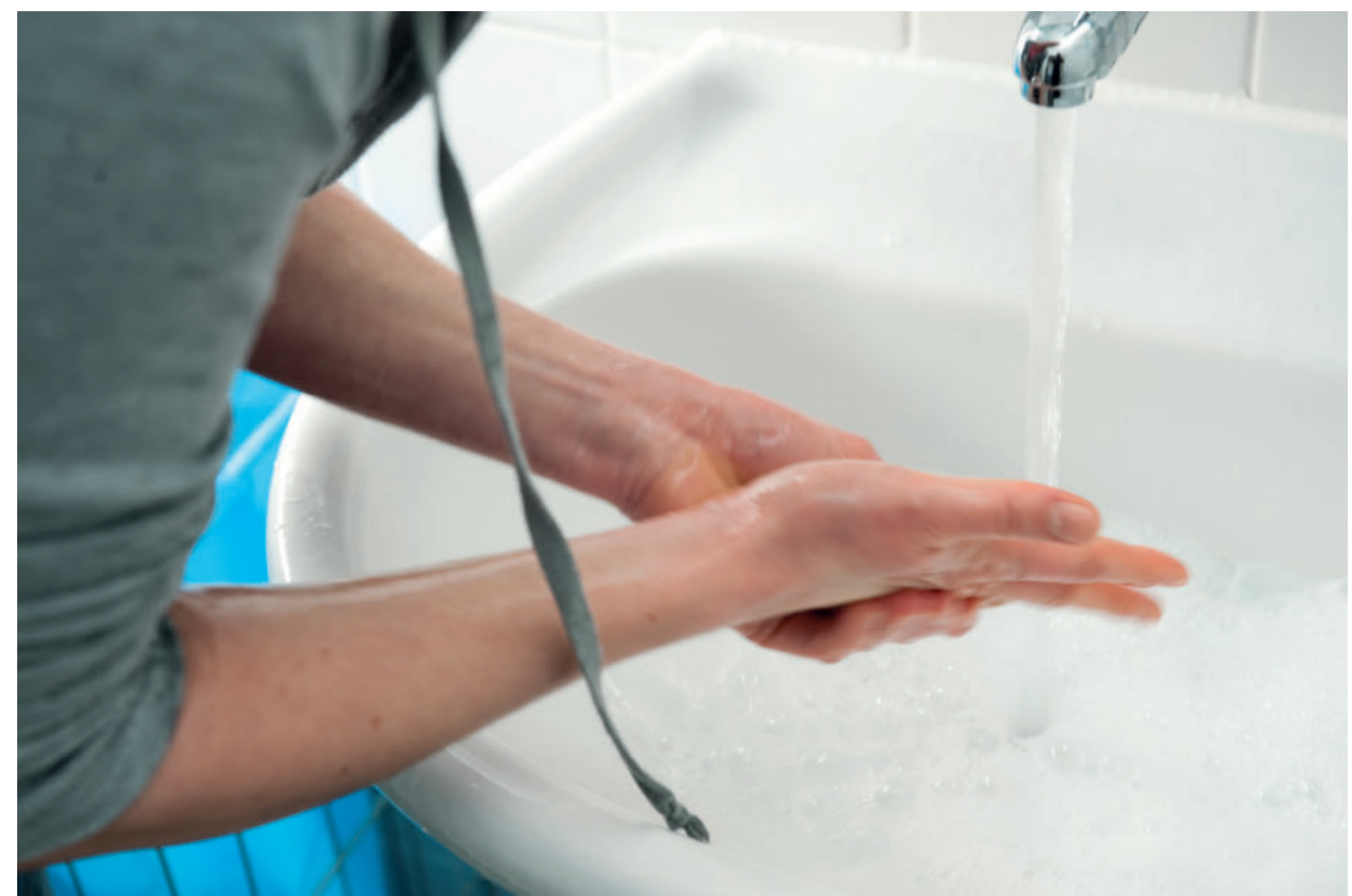

Bei Menschen mit einem Waschzwang kann sich aufgrund der Corona-Pandemie das zwanghafte Verhalten verstärken. (Quelle: Paavo Blåfield/Thieme Gruppe)

„Nicht die Dinge an sich beunruhigen den Menschen, sondern seine Sicht auf die Dinge“ (Epiktet; griechischer Philosoph, 50-138 n. Chr.). Trifft diese Aussage auch in Zeiten von Sars-CoV-2, dem sogenannten neuartigen Coronavirus, zu? Sicherlich gibt es unterschiedliche Umgangsweisen mit dem Virus: von „zu locker“ bis „zu panisch“.
Die Erkrankung, die durch Sars-CoV-2 ausgelöst wird, wird als Covid-19 bezeichnet, die Abkürzung steht für „Corona Virus Disease 2019“. Weltweit verursacht das Virus viele Infizierte und auch Tote und stellt alle Menschen vor enorme Herausforderungen. Das Leben hat sich verändert, wahrscheinlich dauert es Jahre bis sich Wirt- 
schaft, Gesellschaft, Kultur und vieles weitere wieder erholt haben. Kann sich das Leben wieder normalisieren? Was wird als Nächstes kommen? Fragen, die niemand beantworten kann - doch eins steht fest: Das Leben hat sich verändert und wird nicht mehr so sein wie vor Covid-19. Was macht darüber hinaus das Virus mit Menschen, die vor dem Ausbruch der „viralen Bedrohung“ schon durch Ängste in ihrem Leben beeinträchtigt waren? Grundsätzlich ist Angst erst einmal lebenserhaltend, sie kann auch unterstützend und motivierend sein. Denken Sie an die Angst vor einer bevorstehenden Prüfung. Erst wenn diese „Normalität“ aus dem Gleichgewicht gerät, wird der betroffene Mensch zum Sklaven seiner Angst und Hilfe wird notwendig [1]. Das ist der Fall, wenn ein Klient die Wohnung nicht mehr verlassen kann oder ständig seine Angehörigen anruft, um zu wissen, ob es ihnen gut geht. Oder wenn die Angst zum Auslöser für körperliche Symptome, wie Herzrasen oder Schwindelgefühl, wird.

Die große Herausforderung ist es, reale Ängste von pathologischen Ängsten zu unterscheiden. In der „Welt von Corona“ ist dies eine besondere Herausforderung. Denn Realängste sind an Situationen gebunden, die real eine Bedrohung darstellen [1]. Dazu gehört ohne Zweifel Covid19. Und die Angst davor steigt. In einer Anfang April 2020 von Infratest dimap durchgeführten Umfrage [2] zur Angst vor Ansteckung mit dem Coronavirus gaben $17 \%$ der Deutschen an, sehr große Angst zu haben, $34 \%$ hatten große Angst, $35 \%$ weniger große Angst und bei $14 \%$ war die Angst eher klein. Nicht nur Ängste sind in diesem Zusammenhang ein Thema, sondern vieles mehr, wie zum Beispiel die Zwangsstörung. Was macht das Virus mit Menschen mit einer Zwangsstörung? Eine von vielen sinnvollen und wichtigen Schutzmaßnahmen, um das Virus nicht weiterzuverbreiten, ist das gründliche Waschen der Hände für mindestens 30 Sekunden und das Desinfizieren der Hände. Jedoch kann ein Mensch, der seine Hände schon vorher 200-mal am Tag gewaschen hat und mit seinem Therapeuten andere Waschrituale besprochen hat, nun wieder in alte Muster zurückfallen.

\section{Krankheitsbild der Angststörungen}

Zu den Angststörungen gehören laut ICD-10 [4]:

- Agoraphobie mit und ohne Panikstörung,

- soziale Phobie,

- spezifische Phobien,

- Panikstörungen,

- generalisierte Angststörung (betrifft Frau D. aus dem Fallbeispiel),

- Angst und depressive Störung, gemischt.

Im kommenden ICD-11 finden sich folgende Gruppierungen [5]:

- generalisierte Angststörung,

- Panikstörung,

- Agoraphobie,

\section{FALLBEISPIEL GENERALISIERTE ANGSTSTÖRUNG}

Stellen Sie sich folgendes Szenario vor: Frau D. war bis vor 6 Monaten Ihre Klientin. Sie litt über Jahre unter massiver Anspannung und machte sich immer Sorgen um ihre Sicherheit und darüber, dass sie oder ihre Familie erkranken würden und dass sie wirtschaftliche Probleme bekommen würden. Sie konnte kaum schlafen, war oft nervös und telefonierte hinter ihren Mann und ihrer Tochter her. Ständig musste sie sich versichern, dass es ihnen gut geht.

Sie haben mit ihr, entsprechend Ihrem therapeutischen Verfahren, erfolgreich gearbeitet und Frau D. war stabilisiert. Im Abschlussgespräch vor 6 Monaten versicherte sie einen guten Schlaf und ein entspanntes Grundgefühl. Ihren Mann und ihre Tochter ruft sie, wie mit Ihnen vereinbart, 1-mal am Tag an, und damit ist sie zufrieden. Ihre Entspannungsübungen führt sie durch.

Bis jetzt.

Sie ruft heute in Ihrer Praxis an und berichtet, dass alle Symptome wieder da sind, eigentlich ist es jetzt noch schlimmer. Sie verlässt das Haus nicht, aber das sei auch von der Bundesregierung angeordnet. Jedoch läuft sie im Haus hin und her, sie hat Angst, ihre Familie könnte sich jetzt infizieren. Ihre Tochter arbeitet als Krankenschwester, das macht es nicht gerade besser. Wahrscheinlich wird alles noch schlimmer. Wenn sie daran denkt, rast ihr Herz, sie schwitzt und hat Durchfälle. Sie kann kaum noch schlafen und streitet sich immer öfter mit ihrer Tochter, die ihrer Mutter mittlerweile „Telefonterror“ vorwirft. Ihr Mann ist LKW-Fahrer, er geht bei allem Verständnis für seine Frau schon nicht mehr ans Telefon.

\section{Optionen}

- Therapiestunde per Telefon durchführen oder

- Videosprechstunde durchführen (Achtung: Datensicherheit).

Empfehlungen für ein Therapieprogramm bei einer generalisierten Angststörung [3]

- Allgemeine Informationsvermittlung und Selbstbeobachtung.

- Sorgenkonfrontation in sensu und in vivo.

- Kognitive Therapie.

- Angewandte Entspannung.

- spezifische Phobien,

- Trennungsangststörung,

- selektiver Mutismus.

\section{Wie oft treten Angststörung auf?}

Die meisten phobischen Störungen sind häufiger bei Frauen als bei Männern vorhanden ( $\bullet$ Tab. 1).

\section{Welche auslösenden Faktoren gibt es?}

- Im neurobiologischen Bereich liegt eine erhöhte Vulnerabilität vor.

- Eine genetische Disposition wird diskutiert.

- Neurochemische Aspekte spielen eine Rolle bei der Dysfunktionalität von Neurotransmittern. 
- Tab. 1 12-Monats-Prävalenzen von Angststörungen bei 18- bis 79-jährigen [6].

\begin{tabular}{|l|l|l|l|}
\hline Störung (ICD-10-Code) & Frauen in \% & Männer in \% & Anzahl Betroffener in Mio. \\
\hline Angststörung (F40, F41) & 21,4 & 9,3 & 9,8 \\
\hline Panikstörung (mit und ohne Agoraphobie) & 2,8 & 1,2 & 1,3 \\
\hline Agoraphobie & 5,6 & 2,3 & 2,6 \\
\hline Soziale Phobie & 3,6 & 1,9 & 1,7 \\
\hline Generalisierte Angststörung & 3,0 & 1,5 & 1,4 \\
\hline $\begin{array}{l}\text { Spezifische Phobie (Tierphobien, Phobien vor Naturereignissen, } \\
\text { situationale Phobien, Blut-/Spritzen-/Verletzungsphobien) }\end{array}$ & 15,4 & 5,1 & 6,6 \\
\hline
\end{tabular}

- In der Lerntheorie nimmt man an, dass Angst erlernt wird. Dazu gehören:

- Lernen am Modell,

- operantes Lernen,

- klassische Konditionierung.

- Die psychoanalytische Theorie geht von nicht gelösten Konflikten in der Kindheit aus.

\section{Die Formen der Angststörung Agoraphobie [1]}

Die Agoraphobie (altgriechisch agora = Marktplatz) bezeichnet die Platzangst ( $\triangleright$ Tab. 2). Oft wird der Begriff Platzangst fälschlicherweise als Angst in engen Räumen bezeichnet. Es handelt sich jedoch um eine Angst vor weiten Plätzen, die sich auch auf Menschenmengen bezieht. Typische Orte sind auch öffentliche Verkehrsmittel oder Flächen mit großen Menschenmengen. Die Klienten haben Angst vor den oben beschriebenen Orten oder Situationen, sie befürchten, keine Hilfe zu erhalten, falls ihnen etwas geschieht. Diese Angst zeigt sich in plötzlichem Schwindel, Ohnmachtsgefühlen, Depersonalisationsgefühl, Verlust der Blasen- und Darmkontrolle, Herzbeschwerden und kann in eine Panikattacke führen. Zur Diagnosestellung muss die Angst in mindestens 2 der oben beschriebenen Situationen auftreten und darf nicht auf andere Symptome wie Wahn- oder Zwangsgedanken beruhen. Zusätzlich muss der Klient ein Vermeidungsverhalten bezüglich der angstbesetzten Situation zeigen.
Bezug zu Frau D. aus unserem Fallbeispiel: Sie hat keine Angst vor ihr unbekannten Plätzen oder Menschenmengen. Sie würde sie zwar zurzeit meiden, jedoch ist dies eine reale Situation bezogen auf die Vorgaben der Bundesregierung.

\section{Soziale Phobien}

Die soziale Phobie (lateinisch socialis = gesellschaftlich) ist gekennzeichnet durch eine anhaltende Angst vor Situationen, in denen der Klient im Mittelpunkt der Aufmerksamkeit anderer Menschen steht [1]. Ebenfalls muss eine Vermeidungsstrategie beschrieben werden. Die Ängste können sich auch nur in bestimmten Situationen zeigen, zum Beispiel bei Präsentationen oder in Restaurants [1]. Die soziale Phobie ist gekennzeichnet durch eine Angst vor negativen Bewertungen in Situationen, in denen Interaktionen stattfinden oder Leistungen erforderlich sind [7]. Klienten können zu anwesenden Menschen keinen Blickkontakt aufnehmen und befürchten zu erröten. Zusätzlich fangen die Hände an zu zittern, es kann ein Drang zum Wasserlassen gespürt werden. Die Wahrnehmung der körperlichen Reaktion ist wie ein Verstärker, ein Teufelskreis beginnt.

Bezug zu Frau D. aus unserem Fallbeispiel: Die soziale Phobie trifft nicht auf sie zu, sie hat keine Angst vor Aufmerksamkeit.

\section{Spezifische Phobien [1]}

Wie der Name vermuten lässt, handelt es sich um eine Angst vor einem bestimmten angstauslösenden Objekt oder einer spezifischen Situation. Die Störung wird nur diagnostiziert, wenn

- Tab. 2 Überblick über die Formen der Angst [1].

Form der Angst
Agoraphobie mit oder ohne Panikstörung
Soziale Phobie
Spezifische Phobie
Panikstörung
Generalisierte Angststörung
Angst und depressive Störung, gemischt

\section{Symptome}

Angst, in nicht vertrauten Situationen oder Umgebungen hilflos zu sein.

Angst, die Aufmerksamkeit anderer Menschen auf sich zu ziehen. Angst vor einem fest umschriebenen Objekt oder einer bestimmten Situation. Anfallsweises Auftreten der Angstattacken. Anhaltende Angst, die nicht auf spezifische Situationen oder Objekte gerichtet ist. Symptome in leichter Form, ohne vorherrschendes Merkmal einer Angst- oder depressiven Störung. 
die Angst erhebliches Leiden verursacht und die angstauslösende Situation - wann immer möglich - gemieden wird. Am häufigsten treten folgende Ängste auf:

- Akrophobie: Angst vor Höhe;

- Aviophobie: Angst vor dem Fliegen;

- Angst vor Blut;

- Ansteckungsangst;

- Erythrophobie: Angst vor dem Erröten;

- Klaustrophobie: Angst vor dem Aufenthalt in geschlossenen Räumen;

- Zoophobie: Angst vor Tieren.

Bezug zu Frau D. aus unserem Fallbeispiel: Würde Frau D. ausschließlich von einer Ansteckungsfahr berichten und infolgedessen die körperlichen Symptome und einen Leistungsdruck beschreiben, würde dies einer spezifischen Phobie in dieser Form entsprechen.

\section{Panikstörung, episodisch paroxysmale Angst [1]}

Panikattacken sind anfallsartige Angstzustände (griechisch paroxysmal = anfallsweise), die meist nur Minuten andauern, in der Regel circa 10-30 Minuten. Die betroffenen Menschen haben das Gefühl, gleich zu sterben oder verrückt zu werden. Sie ist nicht situationsgebunden und tritt plötzlich mit intensiven vegetativen Symptomen auf, beispielsweise:

- Herzklopfen/beschleunigter Herzschlag (Tachykardie),

- Beklemmungsgefühl,

- Schmerzen in der Brust,

- Hitzewallungen, Schwitzen,

- Erstickungsgefühl, Atemnot,

- Schwindel, Benommenheit, Ohnmachtsgefühle,

- Zittern,

- abdominelle Beschwerden,

- Parästhesien (griechisch para = vorbei; aisthesis= Wahrnehmung, Empfindung),

- Angst vor Kontrollverlust,

- Entfremdungsgefühl,

- Angst, zu sterben.

Die Panikstörung kann mit einem Herzinfarkt verwechselt werden oder umgekehrt.

Bezug zu Frau D. aus unserem Fallbeispiel: Sie hat bis jetzt noch keine Panikattacke erlebt. Ihre Ängste sind nicht anfallsweise, sondern frei flottierend. Sie sind immer vorhanden, intermittieren jedoch in ihrer Intensität.

\section{Generalisierte Angststörung [1]}

Generalisiert steht für Verallgemeinerung, und genau das geschieht bei Menschen mit einer generalisierten Angststörung. Die Angst bezieht sich nicht, wie eine spezifische Angststörung, auf ein bestimmtes Objekt oder eine bestimmte Situation. Sie ist frei flottierend, anhaltend und somit unerträglich. Die Angst hält nicht nur den Klienten in seinen dysfunktionalen Gedanken gefangen, sondern beeinträchtigt ebenfalls das gesamte Umfeld. Die Befürchtung, dass nahestehenden Menschen etwas Schlimmes zu-

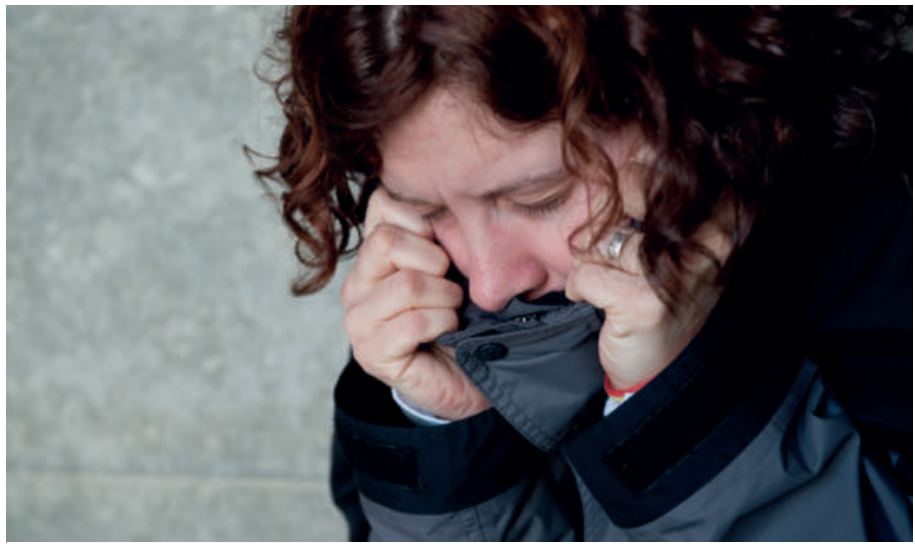

Bei einer Panikattacke haben die Betroffenen das Gefühl, zu sterben oder verrückt zu werden. (Symbolbild/Quelle: Paavo Blåfield/Thieme Gruppe)

stoßen wird, hält den Klienten in Schach. Die Symptome sind an den meisten Tagen in der Woche präsent, mindestens aber über mehrere Wochen bis Monate vorhanden. Folgende Einzelsymptome werden beschrieben:

- Nervosität, Unruhe,

- Konzentrationsstörungen,

- Sorge über zukünftiges Unglück,

- ständiges Gefühl einer Anspannung, Spannungskopfschmerz,

- Schwitzen,

- beschleunigter Herzschlag (Tachykardie),

- beschleunigte Atmung (Tachypnoe),

- abdominelle Beschwerden,

- Schwindelgefühl,

- Mundtrockenheit.

Bezug zu Frau D. aus unserem Fallbeispiel: Bei Frau D. ist die berechtigte reale Angst überschritten. Zudem zeigt sie folgende Symptome:

- Nervosität, Unruhe: Sie läuft im Haus hin und her.

- Sorge über zukünftiges Unglück: Sie hat Angst, ihre Familie könnte erkranken.

- Schwitzen.

- Tachykardie: Ihr Herz rast.

- Abdominelle Beschwerden: Sie hat Durchfall.

- Zusätzlich schläft sie schlecht.

\section{Angst und depressive Störung, gemischt [1]}

Treten gleichzeitig Angst und depressive Symptome auf, kann diese Kategorie verwendet werden. Dies gilt allerdings nur, wenn keine der beiden Störungen so starke Symptome zeigt, dass eine entsprechende einzelne Diagnose gestellt werden muss.

\section{Wie werden Angststörungen diagnostiziert?}

Die diagnostischen Leitlinien sind im ICD-10 beschrieben. Im Vorfeld sollten grundsätzlich somatische Ursachen für 
die auftretenden Symptome durch einen Arzt abgeklärt werden. Die „große Kunst“ ist die Bewertung, ob es noch eine „normale“ oder wann es eine pathologische Angst ist. Wie in unserem Fallbeispiel zum Thema Covid-19 deutlich wird, ist dies nicht ganz einfach. Es stehen verschiedene Testverfahren zur Verfügung. Der Einsatz von Tagebüchern kann für eine Verhaltens- und Situationsanalyse sehr hilfreich sein und eine Diagnose unterstützen. Bekannte Tagebücher sind das Marburger Angsttagebuch [8] oder das Generalisierte Angsttagebuch [9].

\section{Wie verlaufen Angststörungen? [1]}

Der Verlauf von Angststörungen gilt als insgesamt ungünstig. Die Zahlen variieren sehr stark. Die Folgen der Angststörung beeinträchtigen die Lebensqualität maßgeblich. Das kann bis zu vollständigem Rückzug und sozialer Isolation führen sowie im schlimmsten Fall den Verlust des Arbeitsplatzes und/oder der Partnerschaft bedeuten.

\section{Welche Therapieansätze gibt es?}

Die Angst kann nicht durch gutes Zureden beseitigt werden. Die Betroffenen sind sich der „Unsinnigkeit“ ihrer Angst und Gedanken bewusst [1]. Grundsätzlich ist eine vertrauensvolle Beziehung anzustreben, in der offen über die Ängste gesprochen werden kann, ohne dass diese als banal abgestempelt werden [1]. Angst bedeutet grundsätzlich den Verlust von Sicherheit, somit muss die Sicherheit wiederhergestellt werden [1]. An erster Stelle steht also eine Stabilisierung. Die meisten Menschen haben in ihrem Leben bereits Erfolge verzeichnet und verfügen über Fähigkeiten, leider können diese oft nicht mehr gesehen werden. So besitzen Klienten die Fähigkeit, sich Katastrophen sehr gut vorstellen zu können. Wieso wird diese Fähigkeit nicht andersherum genutzt? So können Sie gemeinsam mit den Klienten dysfunktionale Gedanken erarbeiten und in andere Bahnen lenken [1].

Des Weiteren steht eine fachliche Aufklärung im Vordergrund. Der Klient wird über seine Angst informiert. Dazu gehört, sowohl die Angst als auch deren Folgen aufzugeben [1]. Mit dem Klienten wird der genaue Ablauf seiner Stresssituation besprochen. Es muss ihm klar werden, dass nicht das Ereignis alleine die Symptome auslöst, sondern dass die Wahrnehmung dessen die Symptome verstärkt und/oder aufrechterhält. Es werden entsprechende Hausaufgaben besprochen und der Klient übt zwischen den Sitzungen. Geübt werden entsprechende Bewältigungsstrategien, dazu gehören Entspannungsverfahren und das Erlernen von kognitiver Umstrukturierung der dysfunktionalen Gedanken.

Spezielle Therapieverfahren richten sich nach der Ausprägung und der Form der Angst, eventuell ist eine Kombination von pharmakologischer (Aufgabe eines Arztes!) und nichtpharmakologischer Therapie sinnvoll [1].

\section{Psychotherapie [1]}

Die Psychotherapie gliedert sich in verhaltenstherapeutische, tiefenpsychologisch fundierte, psychoanalytische und gesprächstherapeutische Verfahren:

- Zu den angstspezifischen Therapieformen gehört die systematische Desensibilisierung. Dabei wird der Klient anhand einer hierarchischen Angstskala im Zustand der Entspannung schrittweise mit einem angstauslösenden Stimulus konfrontiert. Diese Konfrontation erfolgt zunächst in der Vorstellung, später auch in der Realität (Habituationstraining).

- Als Flooding-Therapie (Reizüberflutung) wird eine Überflutung mit den angstauslösenden Reizen und den dadurch ausgelösten Angstreaktionen bezeichnet. Dabei wird schon zu Beginn der Behandlung - durch in der Angsthierarchie hoch bewertete Auslösesituationen - starke bis maximale Angst provoziert. Der Klient soll erfahren, dass er selbst bei intensivster Angst durch das Aushalten der Situation ein Abklingen der Angst erreichen kann und die Angst „überlebt“.

- Die aufdeckenden tiefenpsychologischen Verfahren versuchen den zugrunde liegenden Konflikt herauszuarbeiten.

- Das Psychodrama ist ursprünglich für Gruppeninterventionen entwickelt worden. Im Rahmen des Psychodramas werden Situationen durchgespielt, um die dabei auftretenden Ängste zugänglich zu machen. Dabei werden angsterzeugende Szenen nachgespielt oder mittels Rollentauschs wiederholt.

\section{Unterstützende Verfahren}

Unterstützende Verfahren sind Entspannungsübungen, wie das autogene Training und die progressive Muskelrelaxation, Atemübungen oder physikalische Maßnahmen. Mit diesen Verfahren kann Einfluss auf das vegetative System genommen werden und sie können somit eine beruhigende und stabilisierende Wirkung haben.

\section{Zwangsstörungen}

Bei einer Zwangsstörung drängen sich in hoher Intensität Gedanken auf, was dazu führen kann, dass Betroffene eventuell das Haus nicht mehr verlassen (weil es ständig kontrolliert werden muss) oder andere Handlungen, zum Beispiel das Händewaschen, nicht unterdrücken können [1].

Unter dem Begriff Zwang werden Handlungen, Handlungsimpulse und Vorstellungen zusammengefasst. Diese drängen sich dem Klienten auf. Er kann sich dagegen nicht wehren. Diese Symptome werden als dem eigenen Ich zugehörig, doch meist als unsinnig und bedrohlich erlebt [1]. Dieses Erleben ist für die Abgrenzung zu anderen Störungen wichtig. Zwangsphänomene beeinträchtigen, wie die Angst, einen Menschen in seinem gesamten Denken, Handeln und sozialen Verhalten [1]. 


\section{Wie oft treten Zwangsstörungen auf?}

Zwangsstörungen stehen an 4. Stelle der häufigsten psychischen Erkrankungen in Deutschland [10]. Der Erkrankungsgipfel liegt zwischen dem 20. und 25. Lebensjahr. Allerdings ist auch im Kindesalter und in späteren Jahren eine Erkrankung möglich. Die Prävalenz liegt bei 1-2\%, aber auch hier sind die Zahlen sehr unterschiedlich und die „Dunkelziffer“ ist hoch. Betroffene Menschen gehen oft erst dann zum Arzt beziehungsweise Therapeuten, wenn der Leidensdruck sehr hoch ist oder das Umfeld reagiert [1]. Zwangssymptome zeigen sich häufig begleitend zu [1]:

- depressiven Störungen,

- Phobien,

- Alkoholmissbrauch,

- Essstörungen,

- Angststörungen.

Die Ursachen der Zwangsstörungen sind den Ursachen der Angststörung gleich zu setzen.

\section{Welche Formen gibt es? \\ Zwangsgedanken}

Zwangsgedanken sind zwanghafte, sich immer wieder aufdrängende, jedoch als unsinnig erkannte Gedanken, zum Beispiel:

- der sich ständig aufdrängende Gedanke, sich im Kontakt mit Menschen und/oder Objekten zu verschmutzen;

- der sich ständig aufdrängende Gedanke, die eigene Gesundheit und/oder die Gesundheit nahestehender Menschen könnte gefährdet sein;

- Angst, bestimmte Dinge getan oder unterlassen zu haben. [1]

Bezug zu Frau D. in unserem Fallbeispiel: Sie berichtet davon, sich ständig Gedanken über die Gesundheit ihrer Familie zu machen. Dieser Punkt müsste bei ihr noch genau analysiert werden.

\section{Zwangsimpulse [1]}

Zwangsimpulse sind zwanghafte, sich gegen den Willen des Klienten aufdrängende Impulse. Sie sind mit einer enormen Angst verbunden, die Handlung könne tatsächlich ausgeführt werden, in den meisten Fällen geschieht dies nicht. Jedoch ist der Impuls so stark, dass der Klient die Angst, diesem Impuls nachzugeben, kaum bewältigen kann. Häufige Inhalte sind:

- aggressive Handlungen anderen Menschen gegenüber;

- sexuelle Impulse, Ausübung an anderen und an sich selbst;

- autoaggressive Handlungen.

\section{Zwangshandlungen [1]}

Zwangshandlungen sind zwanghaft gegen den Willen ausgeführte Handlungen. Bekannt sind der Wasch- und Kontrollzwang. Bei dem Versuch, diese Handlungen zu unterlassen, tritt eine massive innere Spannung auf oder die Angst, wenn diese Handlung nicht durchgeführt wird, ge-

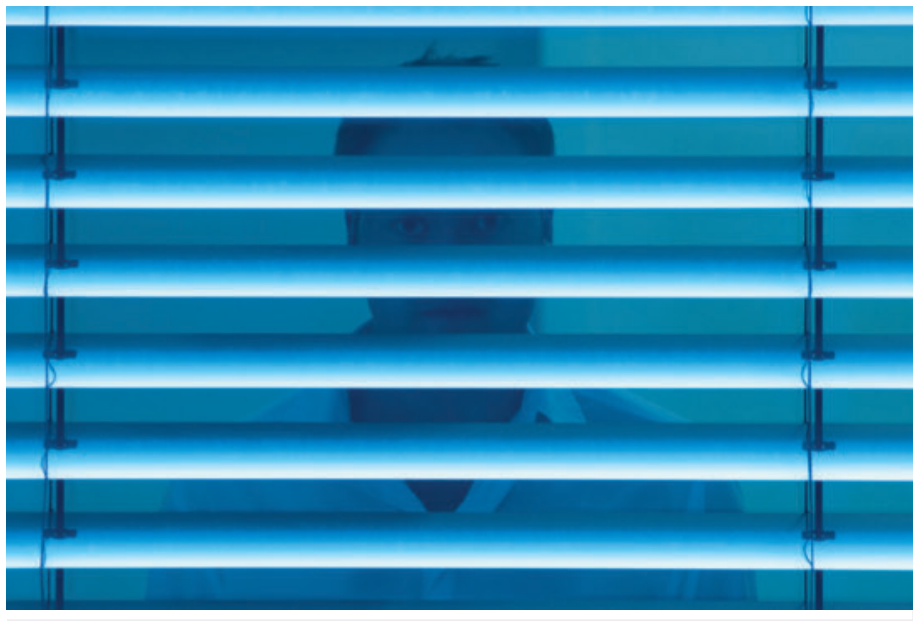

Hilfe wird notwendig, wenn ein Klient beispielsweise die Wohnung nicht mehr verlässt, aus Angst sich anzustecken. (Symbolbild/Quelle: Paavo Blåfield/Thieme Gruppe)

schieht schlimmeres. Um dieser Angst entgegenzuwirken, werden die Handlungen trotz besseren Wissens wieder durchgeführt.

\section{Diagnostische Leitlinien [11]}

Für eine eindeutige Diagnose sollen wenigstens 2 Wochen lang an den meisten Tagen Zwangsgedanken oder -handlungen oder beides nachweisbar sein; sie müssen quälend sein oder die normalen Aktivitäten stören. Die Zwangssymptome müssen folgende Merkmale aufweisen:

- Sie müssen als eigene Gedanken oder Impulse für den Klienten erkennbar sein.

- Wenigstens einem Gedanken oder einer Handlung muss noch, wenn auch erfolglos, Widerstand geleistet werden, selbst wenn sich der Klient gegen andere nicht länger wehren kann.

- Der Gedanke oder die Handlungsausführung dürfen nicht an sich angenehm sein (einfache Erleichterung von Spannung und Angst wird nicht als angenehm in diesem Sinne betrachtet).

- Die Gedanken, Vorstellungen oder Impulse müssen sich in unangenehmer Weise wiederholen.

\section{Wie verlaufen Zwangsstörungen?}

Der Verlauf kann vielgestaltig sein und ist davon abhängig, wie weit sich der Betroffene behandeln lässt. Der Alltag ist oft erheblich eingeschränkt. Ein sozialer Rückzug bis zur totalen Isolation ist nicht selten. [1]

\section{Welche Therapieansätze gibt es?}

Verhaltenstherapeutisch wird eine „Hierarchie“ der Zwänge erstellt. Beim stufenweisen Vorgehen setzt sich der Klient nach und nach seinen Zwängen aus und versucht, ihnen zu widerstehen. Er soll lernen, dass - wenn er die Handlung nicht ausführt - nicht das Befürchtete eintritt. [1] 


\section{Und jetzt zurück auf Start!}

Die $>$ Tab. 3 zeigt die Hauptsorgen und -ängste in Bezug auf die Covid-19/Corona-Pandemie 2020 in Deutschland.

Zur Abgrenzung der Realangst bieten sich Informationen in dieser Form an. Eine Intervention könnte sein, mit dem Klienten sowohl seine persönlichen Ängste als auch die allgemeinen Ängste offen zu thematisieren. Der Klient soll konkret erarbeiten, in welchen Bereichen er sich befindet. Dazu können Sie mit dem Klienten eine Skala von 0-10 erarbeiten: 0 bedeutet keine Angst, 10 bedeutet die stärkste Angst. Welche Zahl würde wohl Frau D. aus unserem Fallbeispiel ankreuzen? Der nächste Weg ist, mit Frau D. zu besprechen, wie wohl andere Menschen ihre Ängste einschätzen würden.

\section{Wie gehen Menschen mit der Realangst um?}

Es werden während der Corona-Pandemie nicht nur Menschen mit vorhandenen Angststörungen zu Ihnen in die Praxis kommen, sondern auch Menschen mit realen Ängsten, die wieder Ihre Stabilität finden möchten und dafür Unterstützung brauchen. Vieles lässt sich zu dem Zeitpunkt, zu dem dieser Artikel entstanden ist (April 2020) noch nicht benennen, die Prognosen bestätigen jedoch diese Theorie.

Eine mögliche Herangehensweise könnte das salutogenetische Konzept von Antonovsky an. Der amerikanisch-israelische Medizinsoziologe Aaron Antonovsky (1923-1994) definierte bereits in den 1980er-Jahren ein Konzept zur Gesunderhaltung [13]. Er entwickelte das sogenannte salutogenetische Konzept und prägte den Begriff der Salutogenese (lateinisch salus = Wohl, Heil, Glück; griechisch genesis $=$ Entstehung) [13] .

Im Mittelpunkt steht die Frage, unter welchen Bedingungen Gesundheit erhalten beziehungsweise wiederhergestellt wird. Antonovsky zufolge wird der Zustand eines Menschen entscheidend durch seine eigene Grundhaltung gegenüber der Welt und sich selbst bestimmt. Diese innere Haltung nennt er Kohärenzgefühl. Salutogenetische Fragestellungen lauten:

- Was erhält den Menschen trotz vieler potenziell belastender/gefährdender Einflüsse gesund?

- Wie schaffen es Menschen, sich von Belastungen/Erkrankungen wieder zu erholen?

- Was ist das Besondere an Menschen, die trotz extremster Belastungen nicht krank werden/stabil bleiben? [14]

Das bedeutet, der Klient kann sich die Antworten auf die Fragen mit Ihnen gemeinsam oder selbstständig erarbeiten. Denn er hat die Krise bereits überwunden, sonst wäre er nicht bei Ihnen in der Praxis. Von den Antworten lassen sich Strategien zur Stabilisierung ableiten. Weiter
- Tab. 3 Was sind Ihre Hauptsorgen oder Bedenken bezüglich der Covid-19/Corona-Pandemie? (Stand: 27.04.2020) [12]

\begin{tabular}{|l|l|}
\hline Ängste & in \% \\
\hline Die Gesundheit meiner Familie & 67 \\
\hline Die wirtschaftliche Stabilität meines Landes & 58 \\
\hline Die Gesundheit meiner Eltern/älterer Freund & 50 \\
\hline Meine körperliche Gesundheit & 50 \\
\hline Meine finanzielle Situation & 36 \\
\hline Die politische Stabilität meines Landes & 26 \\
\hline Mein psychisches Wohlergehen & 23 \\
\hline Meine Arbeitsplatzsicherheit & 21 \\
\hline Lebensmittelknappheit & 18 \\
\hline Unruhen oder Plünderungen & 15 \\
\hline Sonstige & 0 \\
\hline Weiß nicht & 2 \\
\hline
\end{tabular}

postuliert Antonovsky, dass folgende Faktoren erfüllt werden müssen, damit sich der Mensch stabil fühlen kann [15]:

- Gefühl der Verstehbarkeit: Die Fähigkeit, die Umwelt so zu ordnen und zu strukturieren, dass sie verstanden werden kann.

- Gefühl der Machbarkeit: Der Glaube und die Überzeugung, Situationen und Probleme aktiv bewältigen zu können.

- Gefühl der Sinnhaftigkeit: Etwas bewältigen zu wollen, weil es sinnvoll ist beziehungsweise einen Sinn ergibt

Auch daraus lassen sich konkrete Maßnahmen ableiten:

- Gefühl der Verstehbarkeit: Besprechen Sie mit den Klienten fachlich Covid-19. Es gibt sehr viele „Halbweisheiten “ zu dem Virus. Es fängt für viele schon bei den Unterschieden zwischen Bakterium und Virus an. Das Wissen im Umgang bezüglich der Schutzmaßnahmen ist ebenfalls enorm. Selbst die täglichen Nachrichten variieren.

- Gefühl der Machbarkeit: Mit dem Klienten kann erarbeitet werden, was er schon alles in seinem Leben bewältigt hat. Dazu können auch Erkrankungen zählen.

- Gefühl der Sinnhaftigkeit: Bitte stellen Sie nicht die Frage, was das Virus für den Klienten alles Sinnvolles beschert hat. Ein gutes Refraimen kann oft sehr hilfreich sein, jedoch ist es für Menschen, die im schlimmsten Fall einen Angehörigen verloren haben, keine geschickte Intervention. Dieser Bereich kann so umformuliert werden, dass Sie mit dem Klienten erarbeiten, was in dieser Zeit passiert ist und betrachten, ob es für ihn angenehm (positiv) beziehungsweise unangenehm (negativ) ist. 
Eine weitere Möglichkeit, die aus der Resilienz stammt, sind die verschiedenen Ebenen der Selbstfürsorge nach Al Siebert [14]:

- Förderung von Gesundheit und Wohlbefinden: Optionen sind, soziale Netzwerke zu stärken. Vitalisierende Aktivitäten, gerade mit der Familie und/oder Freunden, können stabilisieren.

- Problembewältigung: Welches Problem, welche Herausforderung steht im Vordergrund? Analysieren Sie diese Probleme wie oben beschrieben.

- Innere Torhüter: Dies ist ein sehr wichtiger Punkt zur Stabilisierung. Welche Fähigkeiten besitzt der Klient? Wie ist die Selbstwirksamkeitserwartung? Selbstvertrauen? Selbstwertgefühl? Selbstkonzept?

- Synergie: Was hat der Klient bereits für sich gelernt? Kann er aus der Krise lernen und gestärkt daraus hervor gehen?

- Stärkung: Das Ergebnis sollte sein, aus dem Erlebten gestärkt hervorzugehen, aus der Krise gelernt zu haben.

Egal, mit welcher Intervention Sie mit Ihren Klienten arbeiten, das Ziel sollte sein, dass der Klient das Erlebte in seine Biografie integrieren kann und lernt, damit zu leben. Ihm soll bewusst werden, dass es Vergangenheit ist und eine mögliche Zukunft nicht negativ beeinträchtigen darf.

\section{FAZIT}

Betrachten Sie die Fähigkeiten Ihrer Klienten und vertrauen Sie darauf. Jeder wird durch die Pandemie Veränderungen erfahren, jedoch auch auf „alte“ Erfahrungen zurückgreifen können. Veränderungen wahrzunehmen und zu akzeptieren, gehört zu einem Krisenerleben dazu - jedoch auch zu jeder Therapiesetzung. Verwirklichen Sie mit Ihren Klienten das Ziel, dass Corona ein Teil ihres Lebens ist, dass sie aus dieser Krise Erfahrungen mitnehmen und im besten Fall gestärkt zurückblicken. Nicht die Angst nimmt Überhand, sondern die vorhandene Stärke.

\section{Autorin}

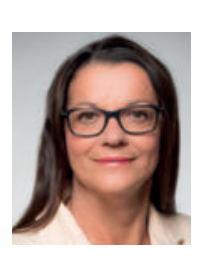

\section{Margarete Stöcker}

Krankenschwester mit Fachweiterbildung Psychiatrie, Diplom-Pflegewirtin, Master of Arts in Gesundheits- und Sozialmanagement, Heilpraktikerin für Psychotherapie, Entspannungspädagogin, Fachbuchautorin, Mimikresonanz-Trainerin E-Mail: m-stoecker@schwerte.de
Literatur

[1] Stöcker M. Praxislehrbuch Heilpraktiker für Psychotherapie. II Krankheitsbilder. 8 Neurotische, Belastungs- und somatoforme Störungen (F40-F48) (I). Stuttgart: Thieme; 2016. DOI: 10.1055/b-0036-137623

[2] Statista. Wie groß ist Ihre Angst vor einer Ansteckung mit dem Coronavirus (COVID-19)? (Stand: 2. April 2020). Im Internet: https://de.statista.com/ (statistik/daten/ studie/1096524/umfrage/umfrage-zur-angst-vor-ansteckung-mit-dem-coronavirus-in-deutschland/); Stand: 29.04.2020

[3] Becker ES, Markgraf J. Generalisierte Angstörung. Ein Therapieprogramm. Weinheim: Beltz PVU; 2002

[4] Deutsches Institut für Medizinische Dokumentation und Information. ICD-10-GM. Im Internet: https://www.dimdi. de/dynamic/de/klassifikationen/icd/icd-10-gm/; Stand: 29.04.2020

[5] World Health Organization. ICD-11. Im Internet: https://icd. who.int/en; Stand: 29.04.2020

[6] Jacobi F, Höfler M, Siegert J et al. Twelve-month prevalence, comorbidity and correlates of mental disorders in Germany: the Mental Health Module of the German Health Interview and Examination Survey for Adults (DEGS1-MH). Int J Methods Psychiatr Res 2014 Sep; 23 (3): 304-19. DOI: 10.1002/ mpr.1439. Epub 2014 Apr 11

[7] Deister A. Angst- und Panikstörungen. In: Möller H, Laux G, Deister A, Hrsg. Psychiatrie, Psychosomatik und Psychotherapie. 5. Auflage. Stuttgart: Thieme; 2013. doi:10.1055/b-002-6253

[8] Margraf J. Panik. Angstanfälle und ihre Behandlung. Heidelberg: Springer; 1990

[9] Hoyer J, Beesdo-Baum K, Becker ES. Ratgeber generalisierte Angststörung. Informationen für betroffene und Angehörige. Göttingen: Hogrefe; 2007

[10] Statista. Häufigste psychische Erkrankungen in Deutschland nach Geschlecht im Jahr 2011. Im Internet: https://de.statista. com/ (statistik/daten/studie/234025/umfrage/haeufigste-psychisch-erkrankungen-in-deutschland-nach-geschlecht/); Stand: 29.04.2020

[11] Dilling, H. Internationale Klassifikation psychischer Störungen: ICD-10 Kapitel V (F). Bern: Huber; 2014

[12] Statista. Was sind Ihre Hauptsorgen oder Bedenken bezüglich der COVID-19/Corona-Pandemie? (Stand: 27.04.2020). Im Internet: https://de.statista.com/ (statistik/daten/ studie/1108157/umfrage/hauptsorgen-und-aengste-wegen-der-covid-19-corona-pandemie/); Stand: 29.04.2020

[13] Antonovsky A. Salutogenese: Zur Entmystifizierung der Gesundheit. Tübingen: dgvt-Verlag; 1997

[14] Stöcker M. Praxislehrbuch Heilpraktiker für Psychotherapie. Stuttgart: Thieme; 2016

[15] Stöcker M. 2016 Praxislehrbuch Heilpraktiker für Psychotherapie. Einführung 2 Wie alles begann. Stuttgart: Thieme; 2016. DOI: 10.1055/b-0036-137616 I

Bibliografie

DOI https://doi.org/10.1055/a-1160-3069

Psych. Pflege Heute 2020; 26: 172-179

(c) Georg Thieme Verlag KG Stuttgart · New York

ISSN 0949-1619 